\title{
Predicting imperfect echo dynamics in many-body quantum systems
}

\author{
Lennart Dabelow and Peter Reimann \\ Fakultät für Physik, Universität Bielefeld, 33615 Bielefeld, Germany
}

(Dated: May 11, 2020)

\begin{abstract}
Echo protocols provide a means to investigate the arrow of time in macroscopic processes. Starting from a nonequilibrium state, the many-body quantum system under study is evolved for a certain period of time $\tau$. Thereafter, an (effective) time reversal is performed that would - if implemented perfectly - take the system back to the initial state after another time period $\tau$. Typical examples are nuclear magnetic resonance imaging and polarization echo experiments. The presence of small, uncontrolled inaccuracies during the backward propagation results in deviations of the "echo signal" from the original evolution, and can be exploited to quantify the instability of nonequilibrium states and the irreversibility of the dynamics. We derive an analytic prediction for the typical dependence of this echo signal for macroscopic observables on the magnitude of the inaccuracies and on the duration $\tau$ of the process, and verify it in numerical examples.
\end{abstract}

\section{INTRODUCTION}

Explaining the irreversibility of processes in macroscopic systems based on the time-reversible laws governing their microscopic constituents is a major task of statistical mechanics, dating all the way back to Boltzmann's $H$-theorem [1] and Loschmidt's paradox [2]. Besides its ontological dimension, this question is also intimately related to the special role of nonequilibrium states and their apparent instability in many-body systems, which generically tend towards equilibrium as time progresses. Characterizing this instability within the realm of quantum mechanics is one goal of the present work. Whereas a direct investigation of the pertinent states (i.e., Hilbert space vectors or density operators) can in principle have academic value, similarly to an analysis of phase-space points in classical systems, we focus here on observable quantities that can be extracted from macroscopic measurements.

Recently, so-called out-of-time-order correlators (OTOCs) have gained considerable attention as a suggestion to generalize concepts from classical chaos theory, notably Lyapunov exponents, to quantum systems [3, 4], but the analogy is far from complete [5, 6]. With this in mind, we follow an even simpler route here and consider so-called echo dynamics [7], where a quantum many-body system with Hamiltonian $H$ starts from a (pure or mixed) "target state" $\rho_{\mathrm{T}}$ and is evolved forward in time for a certain period $\tau$ to reach the "return state" $\rho_{\mathrm{R}}$. At this point, one switches to the inverted Hamiltonian $-H$, so that the direction of time is effectively reversed and the system evolves back towards the target state after another time period $\tau$. By introducing inaccuracies (experimentally unavoidable imprecisions) during this backward evolution via a Hamiltonian of the form $H^{\prime}:=-H+\epsilon V$, the system will not reach the original initial state $\rho_{\mathrm{T}}$ again, but instead approach a perturbed state $\rho_{\mathrm{T}}^{\prime}$ that deviates from it to some extent. In summary,

$$
\rho_{\mathrm{T}} \underset{H}{\stackrel{\tau}{\longrightarrow}} \rho_{\mathrm{R}} \underset{-H+\epsilon V}{\stackrel{\tau}{\longrightarrow}} \rho_{\mathrm{T}}^{\prime}
$$

The deviations between the initial and final states and their dependence on the time span $\tau$ and on the amplitude $\epsilon$ of the considered imperfections then quantify the instability of the nonequilibrium state and the irreversibility of the dynamics.

In case of a pure state, a seemingly direct probe is the Loschmidt echo, i.e., the overlap between the original state $\rho_{\mathrm{T}}$ and the distorted echo state $\rho_{\mathrm{T}}^{\prime}$ [8, 9]. (In case of a mixed state, the corresponding probe is the quantum fidelity.) However, this quantity is practically inaccessible in a many-body system, and, as emphasized above, we will instead concentrate on echoes of macroscopic observables in the following and compare their expectation values at the beginning and at the end of the proposed protocol [9 12]. Since macroscopic observables cannot distinguish between equivalent microstates, one inevitably has to operate in the nonequilibrium regime to be able to characterize the sensitivity of a many-body system towards imperfections.

Taken literally, the suggested protocol (1) can only have the status of a gedankenexperiment since we cannot practically revert the direction of time in a concrete dynamical setup, and also an exchange of the Hamiltonian $H$ for $-H$ is still unphysical in many situations. For example, for a gas of particles in a box, it would require negative particle masses. Nevertheless, it is well known that an effective sign change of the Hamiltonian can be achieved in spin systems, which provides the mechanism underlying spin-echo and nuclear magnetic resonance (NMR) experiments 7]. In fact, the presence of imperfections, i.e., "non-reversed components" of the Hamiltonian, forms the basis of magnetic resonance imaging (MRI) by exploiting that different imperfections in different tissues lead to distinct imperfect echo signals [13]. By means of so-called magic- or polarization-echo techniques, these ideas have also been extended to interacting spin systems [14 21], employing suitably adapted radio-frequency external fields during the "backward" phase of the evolution. More generally, pulse sequences and time-dependent forces have been used to scan quite notable parameter ranges of (effective) spin Hamiltonians in a variety of experimental setups [22 25]. Yet another 
experimental approach towards an effective time reversal consists in tuning a cold atomic gas across a Feshbach resonance [26 28]. Given the neglected unreversed corrections as well as the sophisticated experimental setups necessary, some sort of imperfections of the form considered here are clearly unavoidable and sometimes, like in MRI, even desired.

Furthermore, an alternative view on the suggested protocol may be as follows: For a many-body system with Hamiltonian $\tilde{H}$ ( $=-H$ in the language from above), suppose that we are given an initial state $\rho_{\mathrm{R}}$ (previously the return state), for which it is known that the state obtained after time $\tau, \rho_{\mathrm{T}}=\mathrm{e}^{-\mathrm{i} \tilde{H} \tau} \rho_{\mathrm{R}} \mathrm{e}^{\mathrm{i} \tilde{H} \tau}(\hbar=1)$, is out of equilibrium. Comparing the time evolution from $\rho_{\mathrm{R}}$ under the Hamiltonian $\tilde{H}$ with the dynamics obtained from a perturbed Hamiltonian $\tilde{H}^{\prime}=\tilde{H}+\epsilon V$, we achieve the same effect as in the above echo gedankenexperiment (1). However, there is no need for a "backward Hamiltonian" or some other sort of (experimentally difficult) reversal procedure. From a physical point of view, it is thus irrelevant whether the return state $\rho_{\mathrm{R}}$ was obtained by an explicit unitary time evolution or some other preparation method. Its only relevant property is that it reaches a manifestly out-of-equilibrium state within an accessible time scale $\tau$ in order for the effects of imperfections to become macroscopically visible. This setting is silently included in the following, even though we will employ the language of the echo protocol (11) in the remainder of this work.

In the next Sec. III we set the stage and introduce the suggested echo protocol (11) and the considered imperfections $V$ in detail. Our main result, an analytical prediction characterizing the decay of echo signals under generic time-reversal inaccuracies, is derived in Sec. III. Thereafter, we verify this result numerically in an explicit spin chain model in Sec. [V] In Sec. V] we conclude by summarizing the ideas and relating them to numerical and experimental results from the literature.

\section{SETUP}

To begin with, we represent the Hamiltonian $H$ appearing in (1) in terms of its eigenvalues and eigenvectors as

$$
H=\sum_{n} E_{n}|n\rangle\langle n|
$$

Given some initial state $\rho(0)$ at time $t=0$, the state at any later time $t>0$ follows as $\rho(t):=\mathrm{e}^{-\mathrm{i} H t} \rho(0) \mathrm{e}^{\mathrm{i} H t}$. Being interested in some macroscopic observable (see introduction) in the form of a self-adjoint operator $A$, and denoting its expectation value in an arbitrary state $\rho$ by $\langle A\rangle_{\rho}:=\operatorname{Tr}[\rho A]$, the actual time evolution can thus be written as

$$
\langle A\rangle_{\rho(t)}=\sum_{m, n} \mathrm{e}^{\mathrm{i}\left(E_{n}-E_{m}\right) t}\langle m|\rho(0)| n\rangle\langle n|A| m\rangle .
$$

Despite the quasiperiodic nature of the right-hand side, a many-body system will usually equilibrate 29 31] and spend most of the time close to the time-averaged state $\rho_{\text {eq }}$ with

$$
\left\langle m\left|\rho_{\mathrm{eq}}\right| n\right\rangle:=\delta_{m n}\langle n|\rho(0)| n\rangle,
$$

which we refer to as the equilibrium state in the following.

In order to study the effect of imperfections in terms of expectation values of the observable $A$, the considerations from Sec. Iimply that the system must spend some time away from equilibrium, i.e., there must be a reasonable time interval during which $\langle A\rangle_{\rho(t)}$ differs distinctly from $\langle A\rangle_{\rho_{\mathrm{eq}}}$. Therefore, we focus on these deviations from equilibrium, denoted by the symbol

$$
\mathcal{A}(t):=\langle A\rangle_{\rho(t)}-\langle A\rangle_{\rho_{\mathrm{eq}}} .
$$

Provided that the system is out of equilibrium at time $t=0$, we then ask how special this situation is by investigating how hard it is to return to this state by an effective, but possibly imperfect reversal of time after the system has relaxed for a certain period $\tau$ as detailed in the protocol (11).

In the absence of any imperfections $(\epsilon=0)$, the system traces out the same trajectory in the forward and backward stages, such that

$$
\mathcal{A}(\tau+t)=\mathcal{A}(\tau-t)
$$

for $t \in[0, \tau]$, which constitutes our reference dynamics. It is reasonable to expect that uncontrolled inaccuracies in the time-reversed dynamics will generically push the system closer to equilibrium because they spoil the finetuned correlations between state and observable needed for nonequilibrium conditions. Hence, the backward dynamics will usually lie closer to the equilibrium state than the forward one,

$$
|\mathcal{A}(\tau+t)| \lesssim|\mathcal{A}(\tau-t)| \quad(0 \leq t \leq \tau) .
$$

The sensitivity of the deviations between the perfect and perturbed dynamics with respect to the magnitude $\epsilon$ of the inaccuracies is thus an indicator for the chaoticity and irreversibility of the many-body dynamics. The faster $\mathcal{A}(\tau+t)$ decays with $\epsilon$ compared to $\mathcal{A}(\tau-t)$, the harder it is to design a reversible process and the more extraordinary or special are the nonequilibrium states. Consequently, the relative echo signal $\mathcal{A}(\tau+t) / \mathcal{A}(\tau-t)$ for times $t \in[0, \tau]$ will be our principal object of study in this work, most importantly in the region around the revival or echo peak at $t \approx \tau$, where deviations from equilibrium will be most pronounced.

We denote the time-dependent state of the system in the forward and backward phases by

$$
\begin{aligned}
& \rho_{\mathrm{f}}(t):=\quad \mathrm{e}^{-\mathrm{i} H t} \rho_{\mathrm{T}} \mathrm{e}^{\mathrm{i} H t}, \\
& \rho_{\mathrm{b}}(t):=\mathrm{e}^{\mathrm{i}(H-\epsilon V) t} \rho_{\mathrm{R}} \mathrm{e}^{-\mathrm{i}(H-\epsilon V) t},
\end{aligned}
$$


respectively. We also write $\rho(t)$ to refer to the state during the entire process, i.e., $\rho(t):=\rho_{\mathrm{f}}(t)$ for $t \in[0, \tau]$ and $\rho(t):=\rho_{\mathrm{b}}(t-\tau)$ for $t \in[\tau, 2 \tau]$.

An implicit assumption in all what follows is that the considered many-body system is finite and exhibits a well-defined macroscopic energy $E$. Consequently, the state $\rho(t)$ at any time can only significantly populate energy levels within a macroscopically small energy window

$$
I_{E}:=[E-\Delta E, E]
$$

and the imperfections are assumed to be sufficiently small so that they do not modify this window. In addition, it is taken for granted that the density of states (DOS) of $H$ is approximately constant throughout this energy window, $D_{0} \approx$ const, and that the same holds for the (negative) imperfect backward Hamiltonian $H-\epsilon V$ with eigenvalues $E_{\nu}^{\prime}$ and eigenstates $|\nu\rangle^{\prime}$.

Focusing on the dynamics during the backward phase from (8b), we can use the transformation matrix $U_{\mu k}:=$ ${ }^{\prime}\langle\mu \mid k\rangle$ between the eigenbases of the forward and backward Hamiltonians to write the time-dependent expectation values of the observable $A$ - similarly as in (3) as

$$
\begin{aligned}
\langle A\rangle_{\rho_{\mathrm{b}}(t)}= & \sum_{\mu, \nu}\left\langle\mu\left|\rho_{\mathrm{b}}(t)\right| \nu\right\rangle^{\prime \prime}\langle\nu|A| \mu\rangle^{\prime} \\
= & \sum_{\mu, \nu} \sum_{k, l, m, n} \mathrm{e}^{-\mathrm{i}\left(E_{\nu}^{\prime}-E_{\mu}^{\prime}\right) t} \mathrm{e}^{\mathrm{i}\left(E_{l}-E_{k}\right) \tau} \\
& \quad \times\left\langle k\left|\rho_{\mathrm{T}}\right| l\right\rangle\langle m|A| n\rangle U_{\mu k} U_{\nu l}^{*} U_{\nu m} U_{\mu n}^{*} .
\end{aligned}
$$

Employing the assumed constant DOS for $H$ and $H-\epsilon V$, we can approximately identify energy differences $E_{\nu}^{\prime}-$ $E_{\mu}^{\prime} \simeq E_{\nu}-E_{\mu}$ of the two Hamiltonians within in the relevant time scales [32, 33], so that

$$
\begin{aligned}
\langle A\rangle_{\rho_{\mathrm{b}}(t)}= & \sum_{\mu, \nu} \sum_{k, l, m, n} \mathrm{e}^{-\mathrm{i}\left(E_{\nu}-E_{\mu}\right) t} \mathrm{e}^{\mathrm{i}\left(E_{l}-E_{k}\right) \tau} \\
& \times\left\langle k\left|\rho_{\mathrm{T}}\right| l\right\rangle\langle m|A| n\rangle U_{\mu k} U_{\nu l}^{*} U_{\nu m} U_{\mu n}^{*} .
\end{aligned}
$$

We recall that the Hamiltonian $H$ corresponds to a given many-body quantum system, whereas the perturbation $V$ describes uncontrolled and/or unknown inaccuracies in the time-reversal procedure. In this spirit, we thus model our ignorance about these imperfections by an ensemble of random operators $V$, such that the matrix elements $\langle m|V| n\rangle$ of $V$ in the eigenbasis of $H$ become random variables. The actually considered $V$ ensembles are inspired by the structure of typical perturbations, featuring possible sparsity as well as an interaction strength depending on the energy difference between the coupled states ("bandedness") 34 38]. Requiring Hermiticity, $\langle m|V| n\rangle=\langle n|V| m\rangle^{*}$, and assuming independence of the $\langle m|V| n\rangle$ for $m \leq n$, this suggests the general form

$$
\mathrm{d} P_{m n}(v):=\mathrm{d} \mu_{\left|E_{m}-E_{n}\right|}(v)
$$

for the probability measures of the $\langle m|V| n\rangle$ 's with $m<$ $n$. Here, $\left\{\mathrm{d} \mu_{\Delta}\right\}_{\Delta>0}$ denotes a family of probability measures on $\mathbb{R}$ or $\mathbb{C}$ with mean zero and variance $\sigma_{v}^{2}(\Delta)$, so that the smooth function $\sigma_{v}^{2}(\Delta)$ captures the announced bandedness of the interaction matrix. Likewise, for $m=n$ the probability measure $\mathrm{d} P_{n n}(v):=\mathrm{d} \mu_{0}(v)$ of the (real-valued) diagonal elements $\langle n|V| n\rangle$ is assumed to have vanishing mean (otherwise the perturbation would induce an energy shift) and finite variance.

To obtain a useful prediction regarding the behavior of an actual system, we first compute the average effect of such a perturbation. In a second step, we establish that the resulting prediction satisfies a concentration of measure property, meaning that in a sufficiently highdimensional Hilbert space, a particular realization of the ensemble becomes practically indistinguishable from the average behavior. More precisely, deviations from the average will turn out to be suppressed in the number $N_{v}$ of eigenstates of $H$ that get mixed up by the perturbation $\epsilon V$, to be defined explicitly in Eq. (19) below. Due to the extremely high level density in generic many-body systems, this number $N_{v}$ is typically exponentially large in the system's degrees of freedom $f$ if the perturbation has any appreciable effect at all [39],

$$
N_{v}=10^{\mathcal{O}(f)} \gg 1 \text {. }
$$

\section{RESULTS}

According to Eq. (12), averaging the echo signal over all possible realizations of the $V$ ensemble requires an average over four transformation matrices $U_{\mu k}$, the overlap of the eigenvectors $|k\rangle$ of $H$ and $|\mu\rangle^{\prime}$ of $H-\epsilon V$. These overlaps inherit their distribution from the distribution (13) of the $V$ matrix elements. Writing $\mathbb{E}[\cdots]$ for the average over all $V$ 's, one finds

$$
\begin{aligned}
\mathbb{E}\left[U_{\mu_{1} k_{1}} U_{\mu_{2} k_{2}} U_{\mu_{1} l_{1}}^{*} U_{\mu_{2} l_{2}}^{*}\right]=\delta_{k_{1} l_{1}} \delta_{k_{2} l_{2}} d_{k_{1} k_{2}}^{\mu_{1} \mu_{2}} \\
+\delta_{k_{1} l_{2}} \delta_{k_{2} l_{1}}\left(\delta_{\mu_{1} \mu_{2}} d_{k_{1} k_{2}}^{\mu_{1} \mu_{2}}+f_{k_{1} k_{2}}^{\mu_{1} \mu_{2}}\right)
\end{aligned}
$$

in the limit of sufficiently weak $\epsilon$ [39]. Here

$$
\begin{aligned}
d_{k l}^{\mu \nu} & :=u\left(\alpha \epsilon^{2}, E_{\mu}-E_{k}\right) u\left(\alpha \epsilon^{2}, E_{\nu}-E_{l}\right) \\
f_{k l}^{\mu \nu} & :=-\left(\alpha \epsilon^{2} / 2 \pi D_{0}\right) u\left(\alpha \epsilon^{2}, E_{\mu}-E_{k}\right) u\left(\alpha \epsilon^{2}, E_{\nu}-E_{l}\right)
\end{aligned}
$$

$\times \frac{4 \alpha^{2} \epsilon^{4}+\left(E_{k}-E_{l}\right)^{2}+\left(E_{\mu}-E_{\nu}\right)^{2}-\left(E_{k}+E_{l}-E_{\mu}-E_{\nu}\right)^{2}}{\left[\left(E_{\mu}-E_{l}\right)^{2}+\alpha^{2} \epsilon^{4}\right]\left[\left(E_{\nu}-E_{k}\right)^{2}+\alpha^{2} \epsilon^{4}\right]}$,

and $\alpha:=\pi \bar{\sigma}_{v}^{2} D_{0}$, where $\bar{\sigma}_{v}^{2}$ denotes the mean value of $\sigma_{v}^{2}(\Delta)$, introduced below (13), for small argument. Furthermore, the function $u\left(\alpha \epsilon^{2}, E_{\mu}-E_{k}\right):=\mathbb{E}\left[\left|U_{\mu k}\right|^{2}\right]$ represents the second moment of the transformation matrices $U_{\mu k}$ and is given by the Breit-Wigner distribution

$$
u(\gamma, E)=\frac{\gamma}{\pi D_{0}\left(\gamma^{2}+E^{2}\right)} .
$$

Hence we can identify

$$
N_{v}:=2 \alpha D_{0} \epsilon^{2}=2 \pi \bar{\sigma}_{v}^{2} D_{0}^{2} \epsilon^{2}
$$


as the full width at half maximum of the average overlap $\mathbb{E}\left[\left|U_{\mu k}\right|^{2}\right]$ between eigenvectors of $H$ and $H-\epsilon V$, quantifying the number of energy eigenstates mixed by the perturbation as introduced above Eq. (14).

Exploiting (15) in the average of (12), we obtain

$$
\begin{aligned}
& \mathbb{E}\left[\langle A\rangle_{\rho_{\mathrm{b}}(t)}\right]=\sum_{\mu} d_{k l}^{\mu \mu}\left\langle k\left|\rho_{\mathrm{T}}\right| k\right\rangle\langle l|A| l\rangle+\sum_{\mu, \nu} \mathrm{e}^{-\mathrm{i}\left(E_{\nu}-E_{\mu}\right) t} \times \\
& \times \sum_{k, l}\left[\mathrm{e}^{\mathrm{i}\left(E_{l}-E_{k}\right) \tau} d_{k l}^{\mu \nu}\left\langle k\left|\rho_{\mathrm{T}}\right| l\right\rangle\langle l|A| k\rangle+f_{k l}^{\mu \nu}\left\langle k\left|\rho_{\mathrm{T}}\right| k\right\rangle\langle l|A| l\rangle\right]
\end{aligned}
$$

If we make use of the constant DOS once again (see below (9)), which allows us to shift summation indices, we find that

$$
\begin{aligned}
\sum_{\mu} d_{k l}^{\mu \mu} & =u\left(2 \alpha \epsilon^{2}, \omega_{k l}\right) \\
\sum_{\mu, \nu} \mathrm{e}^{-\mathrm{i}\left(E_{\nu}-E_{\mu}\right) t} d_{k l}^{\mu \nu}= & \mathrm{e}^{\mathrm{i} \omega_{k l} t} \mathrm{e}^{-2 \alpha|t| \epsilon^{2}} \\
\sum_{\mu, \nu} \mathrm{e}^{-\mathrm{i}\left(E_{\nu}-E_{\mu}\right) t} f_{k l}^{\mu \nu}= & -u\left(2 \alpha \epsilon^{2}, \omega_{k l}\right) \mathrm{e}^{-2 \alpha|t| \epsilon^{2}} \\
& \times\left[\cos \left(\omega_{k l} t\right)+\frac{2 \alpha \epsilon^{2}}{\omega_{k l}} \sin \left(\omega_{k l}|t|\right)\right]
\end{aligned}
$$

with $\omega_{k l}:=E_{k}-E_{l}$. Substituting into (20) yields

$$
\mathbb{E}\left[\langle A\rangle_{\rho_{\mathrm{b}}(t)}\right]-\langle A\rangle_{\tilde{\rho}}=\mathrm{e}^{-2 \alpha|t| \epsilon^{2}}\left[\langle A\rangle_{\rho_{\mathrm{f}}(\tau-t)}-\langle A\rangle_{\tilde{\rho}}\right]+R(|t|)
$$

with the locally averaged equilibrium state $\tilde{\rho}$ given by $\langle m|\tilde{\rho}| n\rangle:=\delta_{m n} \sum_{k} u\left(2 \alpha \epsilon^{2}, E_{n}-E_{k}\right)\left\langle k\left|\rho_{\mathrm{T}}\right| k\right\rangle$ and

$$
\begin{array}{r}
R(t):=\mathrm{e}^{-2 \alpha t \epsilon^{2}} \sum_{k, l}\left\langle k\left|\rho_{\mathrm{T}}\right| k\right\rangle\langle l|A| l\rangle u\left(2 \alpha \epsilon^{2}, \omega_{k l}\right) \\
\times\left\{1-\cos \left(\omega_{k l} t\right)-\frac{2 \alpha \epsilon^{2}}{\omega_{k l}} \sin \left(\omega_{k l} t\right)\right\} .
\end{array}
$$

Note that $\tilde{\rho}$ is the state approached for large times and can thus be identified with the equilibrium state $\rho_{\text {eq }}$ from (5). It usually corresponds to the pertinent thermal state 41 44]. We also observe that $R(t)$ vanishes at $t=0$ and for $t \rightarrow \infty$. Furthermore, as shown in Appendix A, its magnitude can be bounded from above for arbitrary $t \geq 0$ according to

$$
R^{2}(t) \leq \frac{\delta^{2} A}{50 N_{v}}
$$

where $N_{v}$ is the width of the eigenvector overlaps from (19), and

$$
\delta^{2} A:=\sum_{n: E_{n} \in I_{E}}\left(A_{n n}-\langle A\rangle_{\rho_{\mathrm{mc}}}\right)^{2}
$$

where $\rho_{\mathrm{mc}}$ denotes the microcanonical density operator corresponding to the energy window from (9). For generic (non-integrable) Hamiltonians $H$, essentially all observables $A$ of actual interest are expected to satisfy the so-called eigenstate thermalization hypothesis (ETH) [45]. Hence, the right-hand side of (27) can be roughly estimated as $\|A\|^{2}$ (with $\|A\|$ denoting the operator norm of $A$, i.e., the largest eigenvalue in modulus) and $R(t)$ in (24) can be neglected according to (14) and (26).

For integrable systems, the relevant observables are still expected to satisfy the so-called weak ETH [46 48], and thus the right hand side of (27) can be roughly estimated as $\|A\|^{2} N / f$, where $N$ is the number of energy levels $E_{n}$ contained in $I_{E}$, while $f$ counts the degrees of freedom of the considered system and therefore scales as $\ln (N)$ (see also (14)). Again, one can conclude from (26) that $R(t)$ in (24) amounts in many cases to a small correction.

Finally, we point out that the bound $(26)$ is still rather loose since the oscillating character of the summands in (25) with respect to both $\omega_{k l}$ and $t$ will usually result in very strong "accidental cancellation" effects, which are entirely disregarded in our derivation of the "worst case" bound (26) in Appendix A

Indeed, we have not been able to identify any specific example of practical interest where the last term in (24) plays a significant role. Accordingly, this term is henceforth considered as negligible. With (5), (8), and (14) we thus arrive at the first key result of this section,

$$
\frac{\mathbb{E}[\mathcal{A}(\tau+t)]}{\mathcal{A}(\tau-t)}=\mathrm{e}^{-2 \alpha t \epsilon^{2}} \quad(0 \leq t \leq \tau),
$$

which quantifies the average effect of imprecisions during the backward evolution within the considered ensembles of $V$ 's and for small enough $\epsilon$. Analogously to Ref. [39], one can then proceed to derive a bound for the variance of $\mathcal{A}(\tau+t)$. This leads to

$$
\mathbb{E}\left[\mathcal{A}(\tau+t)^{2}\right]-\mathbb{E}[\mathcal{A}(\tau+t)]^{2} \leq \frac{C_{v}\|A\|^{2}}{N_{v}},
$$

where $C_{v}$ is a constant of order $10^{3}$ or less. In view of (14), the variance (29) of $\mathcal{A}(\tau+t)$ with $V$ is thus exponentially small in the number of degrees of freedom, establishing a so-called concentration of measure property of the considered ensembles of $V$ operators. For instance, invoking Chebyshev's inequality from probability theory, the estimate (29) implies that the probability for $\mathcal{A}(\tau+t)$ to differ by more than $\|A\| / N_{v}^{1 / 3}$ from the average $\mathbb{E}[\mathcal{A}(\tau+t)]$ at a certain instant in time $t$ is less than $C_{v} / N_{v}^{1 / 3}$. This is our second key result of this section, promoting Eq. (28) from a mere statement about the ensemble average to a prediction for individual realizations: Since deviations from the average are extremely rare for reasonably large many-body systems, we can conclude that

$$
\frac{\mathcal{A}(\tau+t)}{\mathcal{A}(\tau-t)}=\mathrm{e}^{-2 \alpha t \epsilon^{2}} \quad(0 \leq t \leq \tau)
$$

is an excellent approximation for the vast majority of time-reversal inaccuracies $V$ captured by the considered 


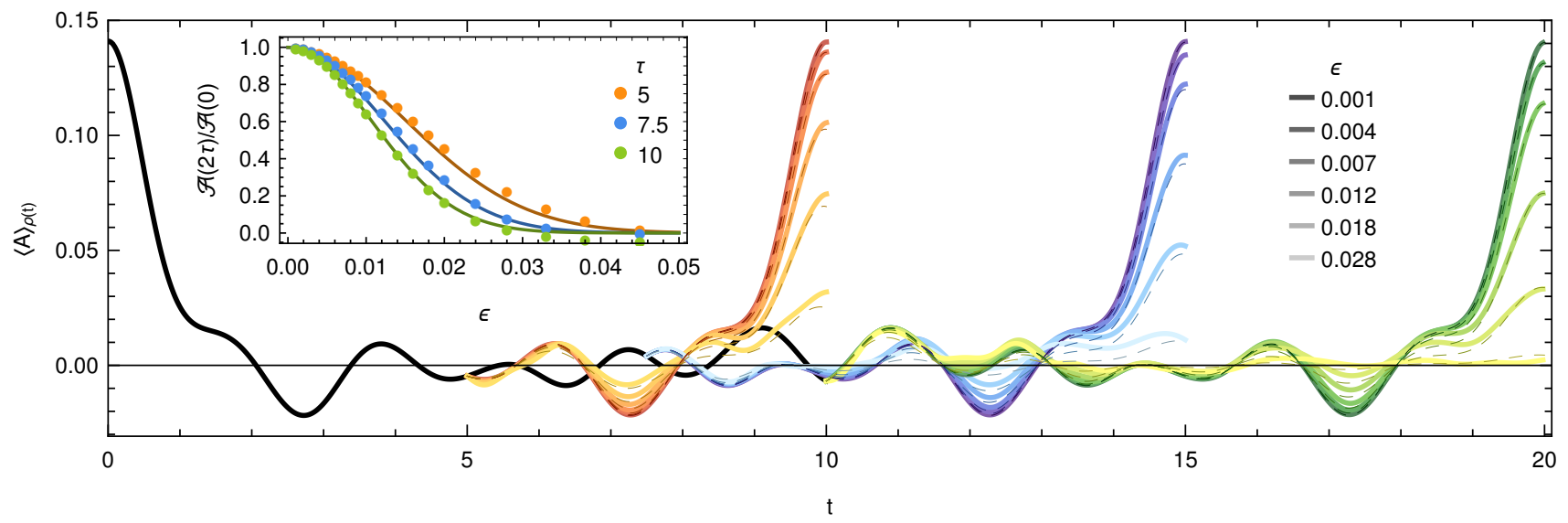

FIG. 1: Time-dependent expectation values of the staggered magnetization (33) for the spin- $\frac{1}{2}$ XXX chain (31) with $L=14$ under the "imperfect reversal" protocol (1) with various perturbation strengths $\epsilon$ and reversal times $\tau$. The initial condition is chosen as a filtered Néel target state $\rho_{\mathrm{T}}=|\psi\rangle\langle\psi|$ according to Eq. (34) with $\sigma_{\psi}=1.3$. The imperfection Hamiltonian $V$ in (1) is of the "spin-glass" form (32). Solid lines correspond to the numerical results using exact diagonalization. Dashed lines show the prediction for the backward (echo) dynamics according to Eqs. (30) and (35). Time reversal is initiated after time $\tau=5$ (red-toned curves), $\tau=7.5$ (blue-toned), or $\tau=10$ (green-toned). Inset: Ratio $\mathcal{A}(2 \tau) / \mathcal{A}(0)$ of the echo peak height (at time $t=2 \tau)$ and the initial value (at $t=0$ ) as a function of the perturbation strength $\epsilon$ for the different reversal times $\tau$. Data points are the numerical solutions, solid lines are the analytical prediction from (30) and (35).

ensembles. This relation for the echo dynamics (1) under an imperfect backward Hamiltonian constitutes our main result. It asserts that the echo signal is exponentially suppressed in the propagation time $t$ and the intensity $\epsilon^{2}$ of the imperfections.

\section{EXAMPLE}

We consider a spin- $\frac{1}{2}$ XXX chain model with Hamiltonian

$$
H=-\sum_{i=1}^{L-1} \boldsymbol{\sigma}_{i} \cdot \boldsymbol{\sigma}_{i+1},
$$

where $\boldsymbol{\sigma}_{i}=\left(\sigma_{i}^{x}, \sigma_{i}^{y}, \sigma_{i}^{z}\right)$ is a vector of Pauli matrices acting on site $i$. For the perturbation $V$, we choose

$$
V=\sum_{i<j} \sum_{\alpha, \beta=1}^{3} J_{i j}^{\alpha \beta} \sigma_{i}^{\alpha} \sigma_{j}^{\beta},
$$

where the couplings $J_{i j}^{\alpha \beta}$ are drawn independently from a normal distribution (unbiased Gaussian with unit variance). As the observable, we take the staggered magnetization

$$
A=\frac{1}{L} \sum_{i=1}^{L}(-1)^{i} \sigma_{i}^{z} .
$$

Turning to the initial (target) state $\rho_{\mathrm{T}}=|\psi\rangle\langle\psi|$, let us first consider a Néel state of the form $|\bar{\psi}\rangle:=|\downarrow \uparrow \downarrow \uparrow \cdots\rangle$. In order to account for the requirement that the density of states should be approximately constant (see below
Eq. (9)), we rescale the probability amplitudes $\langle n \mid \bar{\psi}\rangle$ according to the corresponding energy eigenvalues $E_{n}$ with a Gaussian weight of zero mean and standard deviation $\sigma_{\psi}$, resulting in

$$
|\psi\rangle:=C \sum_{n} \mathrm{e}^{-E_{n}^{2} / 2 \sigma_{\psi}^{2}}\langle n \mid \bar{\psi}\rangle|n\rangle,
$$

where the normalization constant $C$ is chosen such that $\langle\psi \mid \psi\rangle=1$. As discussed around Eq. (9), the large isolated systems we have in mind (e.g., an MRI sample) are expected to exhibit a macroscopically well-defined energy, so that $\sigma_{\psi}$ should lie below the measurement resolution. In particular, while it is possible to prepare a clean Néel state in few-body experiments with cold atoms, such attempts will most likely result in a filtered or coarsegrained variant as the degrees of freedom increase.

Quantitatively, for the example in Fig. 1 we chose a chain length of $L=14$ and a standard deviation of $\sigma_{\psi}=1.3$, so that the state (34) is focused around energy $E=0$ with about $15 \%$ of the total $2^{L}=16384$ levels $E_{n}$ within $\pm \sigma_{\psi}$. This procedure reduces the staggered magnetization $A$ of $|\psi\rangle$ compared to $|\bar{\psi}\rangle$, but still gives an appreciably ouf-of-equilibrium expectation value (see also Fig. 1).

After diagonalizing $H$ numerically, we estimated the density of states $D_{0}$ by averaging over all states with energies $E_{n} \in\left[-2 \sigma_{\psi}, 2 \sigma_{\psi}\right]$ (receiving about $95 \%$ of the weight), resulting in $D_{0} \approx 962$. Furthermore, we extracted $\bar{\sigma}_{v}^{2}$ [see below Eq. (17)] from the squared matrix elements $|\langle m|V| n\rangle|^{2}$ within the relevant energy window in (9) (determined again by the $2 \sigma_{\psi}$ criterion) by way of averaging around the diagonal within a band of 1000 states, yielding $\bar{\sigma}_{v}^{2} \approx 0.0729$. According to the definition 
below Eq. (17) this yields

$$
\alpha=\pi \bar{\sigma}_{v}^{2} D_{0} \simeq 220 .
$$

All parameters entering our analytical prediction (30) are thus explicitly available, i.e., there is no free fit parameter.

In Fig. 1, we compare the numerical results obtained by exact diagonalization with our prediction (30) for different propagation times $\tau$ and perturbation strengths $\epsilon$, showing good agreement. The largest deviations become apparent for small $\tau$ and large $\epsilon$. By generalizing the analysis of Ref. [39], we expect that the band profile $\sigma_{v}^{2}(\Delta)$ of the perturbation $V$ becomes important in this regime. The exponential form (30) for the suppression of the echo signal is then anticipated to show a transition to a Bessel-like decay of the form $4 J_{1}(x)^{2} / x^{2}$, where $x$ depends linearly on the noise strength $\epsilon$, the reversal time $\tau$, and the square root of the band width. More generally, within the considered ensemble of inaccuracies the echo-signal attenuation is essentially given by the Fourier transform of $u(\gamma, E)$ introduced above Eq. (18), which can be viewed as the ensemble-averaged fidelity or survival probability [9, 40] of an eigenstate $|n\rangle$ of the clean Hamiltonian $H$ under the imprecise backward Hamiltonian $H^{\prime}$. When the influence of the random inaccuracies in $H^{\prime}$ increases, the echo signal may therefore be expected to approach the known decay of fidelity in pure random matrix models [40]. Unfortunately, we are not aware of an exact analytic solution of the pertinent equations in the intermediate regime between the exponential and Bessel-type behaviors [39].

\section{CONCLUSIONS}

We investigated the stability of observable echo signals in many-body quantum systems under the influence of uncontrolled imperfections in the pertinent effective time reversal. The considered protocol starts from a nonequilibrium initial state and lets the system evolve under the time-independent Hamiltonian $H$ for some time $\tau$, at which an (effective) time reversal is performed that directs the system back towards the initial nonequilibrium state. By introducing small inaccuracies in the timereversed Hamiltonian, we obtained a measure for the instability of nonequilibrium states and the irreversibility of the dynamics in terms of directly observable quantities.

Our prediction for the relative echo signal under such a distorted backward Hamiltonian, Eq. (30), includes an exponential dependence on both the squared perturbation strength $\epsilon^{2}$ and the propagation time $t$. In particular, the height of the echo peak at $t \approx \tau$ is thus expected to decay exponentially in $\tau$. Systems with this property were labeled "irreversible" in Ref. [11], as opposed to "reversible" ones where the decay is (at most) algebraic. In this sense, one may interpret the present result as a prediction that many-body quantum systems are typically irreversible. However, it should be pointed out that the functional dependence of the echo peak on $\tau$ rather appears to be a property of the inaccuracies $V$ than of the system itself, even though the structure of $V$ is (in a real system) of course influenced by the properties of the system. In any case, this functional dependence could also be confirmed for an exemplary spin- $\frac{1}{2}$ XXX model, whose dynamics shows good agreement with our analytical prediction without any remaining fit parameter.

The paradigmatic example of macroscopic echo experiments are spin echoes and NMR [7], where nuclear spins precess in a strong magnetic field at different frequencies due to local inhomogeneities, leading to dephasing of the initially aligned magnetic moments. Applying a $\pi$ pulse at time $\tau$ reverses the relative orientation between the spins and the external field, and thus effectively changes the sign of the corresponding term in the pertinent model Hamiltonian. However, interactions among the spins and with the environment are not reversed and amount to a "perturbation" that causes deviations of the echo signal at time $2 \tau$, which - in line with our general echo analysis here - typically decays exponentially with $\tau$. It should be noted that the "imperfections" in this context are usually vital in applications like MRI, precisely because different imperfections lead to slightly different decay rates and therefore allow one to distinguish different materials.

Experimental implementations of echo protocols are also available in a variety of interacting spin systems, see, e.g., Refs. [15 17, 20, 21]. In these experiments, an effective sign flip of the dominant part of the Hamiltonian (including dipole-dipole or even quadrupole interactions) is achieved by means of an elaborate application of radio-frequency magnetic fields. The prevailing inaccuracies leading to deviations from the perfectly reversed signal are again due to nonreversible correction terms in the Hamiltonian as well as possible experimental imprecisions in carrying out the required protocol. Their major contribution is thus expected to be of the type studied here, too.

Specifically, the experimental study [20] indeed reports an exponential decay of the peak height with the reversal time $\tau$ in a polarization echo experiment involving the nuclear spins of a cymantrene polycrystalline sample. In the same study, data obtained from a ferrocene sample suggest an approximately Gaussian-shaped dependence, see also Ref. 21]. The authors explain this with the much larger relative strength of the nonreversible component in the Hamiltonian, compatible with our prediction that a crossover from an exponential to a Bessel-like [49] decay is expected as the relative strength of $V$ increases, see the discussion at the end of Sec. IV]

From a conceptual point of view, the approach of our present work, where the Hamiltonians of the forward and backward phases differ slightly, assesses (via observable quantities) the stability of many-body trajectories with respect to variations of the dynamical laws. Therefore, it should be no surprise that deviations grow with the propagation time $\tau$, and the exponential dependence might 
have been anticipated from perturbation-theoretic considerations, even though the applicability of standard perturbation theory is rather limited for typical manybody systems with their extremely dense energy spectra. In that sense, the present derivation by nonperturbative methods is reassuring and also indicates how deviations from the exponential behavior will manifest themselves if the influence of imperfections increases. For future work, it will be interesting to investigate a complementary approach that studies the sensitivity towards variations of the initial conditions in macroscopic quantum system.

\section{Acknowledgments}

L. D. thanks Patrick Vorndamme for inspiring discussions. This work was supported by the Deutsche Forschungsgemeinschaft (DFG) within the Research Unit FOR 2692 under Grant No. 397303734 and by the Paderborn Center for Parallel Computing $\left(\mathrm{PC}^{2}\right)$ within the Project HPC-PRF-UBI2.

\section{Appendix A: Derivation of Eq. 26}

Exploiting Eq. (18) we rewrite $R(t)$ from (25) as

$$
\begin{aligned}
R(t) & =\tilde{R}\left(2 \alpha \epsilon^{2} t\right), \\
\tilde{R}(t) & :=\sum_{m, n}\left\langle m\left|\rho_{\mathrm{T}}\right| m\right\rangle\langle n|A| n\rangle h(m-n, t), \\
h(n, t) & :=\frac{e^{-t}}{\pi N_{v}} \frac{1-\cos \left(t n / N_{v}\right)-\frac{\sin \left(t n / N_{v}\right)}{n / N_{v}}}{1+\left(n / N_{v}\right)^{2}},
\end{aligned}
$$

where $N_{v}$ is defined in (19), and where $\omega_{k l}:=E_{k}-E_{l}$ (see below Eq. (21) ) has been approximated by $(k-l) / D_{0}$, as justified below Eq. (9). that

According to (14), we can and will take for granted

$$
N_{v} \gg 1
$$

In view of it follows that the sum $\sum_{n} h(n, t)$ can be very well approximated by the integral $\int \mathrm{d} x h(x, t)$. Moreover, a straightforward but somewhat tedious calculation yields $\int \mathrm{d} x h(x, t)=0$. Therefore, we can subtract a constant value from the observable $A$ without changing the value of $\tilde{R}(t)$ in A2 . By means of the definition

$$
\tilde{A}_{n n}:=\langle n|A| n\rangle-\langle A\rangle_{\rho_{\mathrm{mc}}}
$$

we thus can rewrite $\mathrm{A2}$ as

$$
\begin{aligned}
\tilde{R}(t) & =\sum_{m}\left\langle m\left|\rho_{\mathrm{T}}\right| m\right\rangle \tilde{Q}(m, t), \\
\tilde{Q}(m, t) & :=\sum_{n} \tilde{A}_{n n} h(m-n, t) .
\end{aligned}
$$

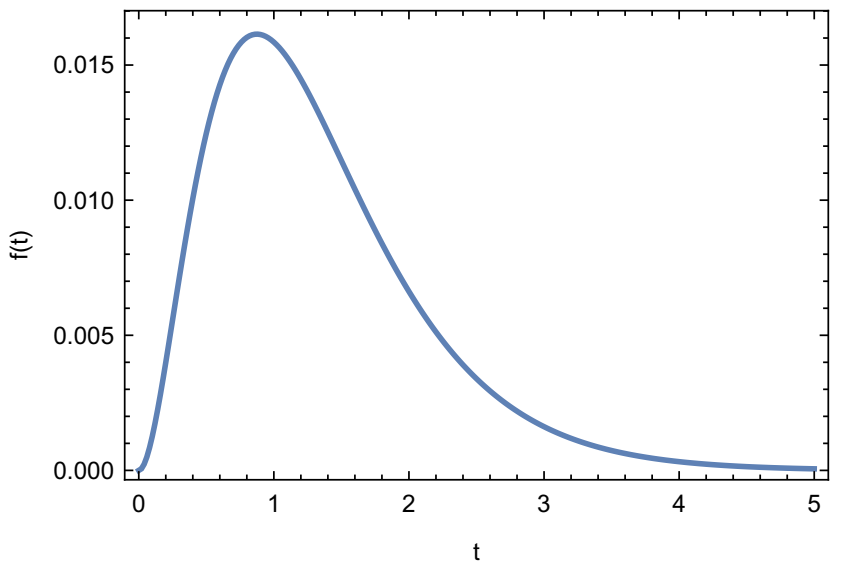

FIG. 2: Numerical evaluation of the function $f(t)$ from (A14).

Exploiting the Cauchy-Schwarz inequality yields

$$
\tilde{Q}^{2}(m, t) \leq\left[\sum_{n}\left(\tilde{A}_{n n}\right)^{2}\right]\left[\sum_{n} h^{2}(m-n, t)\right] .
$$

Since the last sum over $n$ is independent of $m$ it follows that

$$
\begin{aligned}
|\tilde{Q}(m, t)| & \leq \sqrt{Q(t)} \\
Q(t) & :=q(t) \sum_{n}\left(\tilde{A}_{n n}\right)^{2} \\
q(t) & :=\sum_{n} h^{2}(n, t) .
\end{aligned}
$$

Accordingly, $\tilde{R}(t)$ from A6 can be upper bounded as

$$
|\tilde{R}(t)| \leq \sqrt{Q(t)} \sum_{m}\left\langle m\left|\rho_{\mathrm{T}}\right| m\right\rangle=\sqrt{Q(t)}
$$

where we exploited that $\rho_{\mathrm{T}}$ is a positive semi-definite operator of unit trace.

Due to (A3) and (A4) one can conclude - similarly as below (A4) - that the sum on the right hand side of (A11) is very well approximated by the integral $\int \mathrm{d} y h^{2}(y, t)$. After going over from the integration variable $y$ to $x:=$ $y / N_{v}$ one thus obtains in very good approximation

$$
\begin{aligned}
q(t) & =N_{v}^{-1} f(t) \\
f(t) & :=e^{-2 t} \int \mathrm{d} x\left[\frac{1-\cos (x t)-\sin (x t) / x}{\pi\left[1+x^{2}\right]}\right]^{2} .
\end{aligned}
$$

An analytical evaluation of $f(t)$ from (A14 is possible but quite arduous, while a numerical evaluation is straightforward, see Fig. 2. In either case, one finds that

$$
0 \leq f(t) \leq 1 / 50=: c
$$

for all $t \geq 0$. Taking into account (A1), (A10), A12, and (A13), we thus arrive at

$$
R^{2}(t) \leq \frac{c}{N_{v}} \sum_{n}\left(\tilde{A}_{n n}\right)^{2} .
$$


As discussed above Eq. (9), the diagonal matrix elements $\langle n|\rho(0)| n\rangle$ appearing in (3) vanish whenever $E_{n} \notin$ $I_{E}$. Accordingly, we can arbitrarily modify the corresponding $\langle n|A| n\rangle$ 's without any further consequences in (3). Specifically, we can modify them so that all $\tilde{A}_{n n}$ in
(A5) are zero if $E_{n} \notin I_{E}$. Therefore, the summation on the right hand side of (A16) can be restricted to those $n$ with $E_{n} \in I_{E}$. Altogether, we thus recover (26) and (27) from the main text.
[1] L. Boltzmann, Weitere Studien über das Wärmegleichgewicht unter Gasmolekülen, Sitzungsberichte Akad. Wiss. Wien 66, 275 (1872); reprint: Further Studies on the Thermal Equilibrium of Gas Molecules, The Kinetic Theory of Gases, 262 (World Scientific, 2003).

[2] J. Loschmidt, Über den Zustand des Wärmegleichgewichtes eines Systems von Körpern mit Rücksicht auf die Schwerkraft, Sitzungsberichte Akad. Wiss. Wien 73, 128 (1876).

[3] J. Maldacena, S. H. Shenker, D. Stanford, A bound on chaos, J. High Energy Phys. 2016, 106 (2016).

[4] B. Swingle, Unscrambling the physics of out-of-timeorder correlators, Nat. Phys. 14, 988 (2018).

[5] Q. Hummel, B. Geiger, J. D. Urbina, and K. Richter, Reversible Quantum Information Scrambling in Many-Body Systems near Criticality, Phys. Rev. Lett. 123, 160401 (2019).

[6] S. Pilatowsky-Cameo, J. Chávez-Carlos, M. A. Bastarrachea-Magnani, P. Stránský, S. LermaHernández, L. F. Santos, and J. G. Hirsch, Positive quantum Lyapunov exponents in experimental systems with a regular classical limit, Phys. Rev. E 101, 010202(R) (2020).

[7] E. L. Hahn, Spin Echoes, Phys. Rev. 80, 580 (1950).

[8] A. Peres, Stability of quantum motion in chaotic and regular systems, Phys. Rev. A 30, 1610 (1984).

[9] T. Gorin, T. Prosen, T. H. Seligman, and M. Žnidarič, Dynamics of Loschmidt echoes and fidelity decay, Phys. Rep. 435, 33 (2006).

[10] B. V. Fine, T. A. Elsayed, C. M. Kropf, and A. S. de Wijn, Absence of exponential sensitivity to small perturbations in nonintegrable systems of spin 1/2, Phys. Rev. E 89, 012923 (2014).

[11] M. Schmitt and S. Kehrein, Effective time reversal and echo dynamics in the transverse field Ising model, EPL 115, 50001 (2016).

[12] M. Schmitt and S. Kehrein, Irreversible dynamics in quantum many-body systems, Phys. Rev. B 98, 180301 (2018).

[13] M. T. Vlaardingerbroek and J. A. den Boer, Magnetic Resonance Imaging - Theory and Practice, Third Edition, Springer (Berlin, Heidelberg, 2003).

[14] H. Schneider and H. Schmiedel, Negative time development of a nuclear spin system, Phys. Lett. 30A, 298 (1969).

[15] W.-K. Rhim, A. Pines, and J. S. Waugh, Violation of the spin-temperature hypothesis, Phys. Rev. Lett. 25, 218 (1970).

[16] W.-K. Rhim, A. Pines, and J. S. Waugh, Time-Reversal Experiments in Dipolar Coupled Spin Systems, Phys. Rev. B 3, 684 (1971).

[17] S. Zhang, B. H. Meier, and R. R. Ernst, Polarization echoes in NMR, Phys. Rev. Lett. 69, 2149 (1992).
[18] R. Kimmich, J. Niess, and S. Hafner, Quadrupolar magic echoes, Chem. Phys. Lett. 190, 503 (1992).

[19] S. Hafner, D. E. Demco, and R. Kimmich, Magic echoes and NMR imaging of solids, Solid State Nucl. Mag. Res. 6, 275 (1996).

[20] P. R. Levstein, G. Usaj, and H. M. Pastawski, Attenuation of polarization echoes in nuclear magnetic resonance: A study of the emergence of dynamical irreversibility in many-body quantum systems, J. Chem. Phys. 108, 2718 (1998).

[21] G. Usaj, H. M. Pastawski, and P. R. Levstein, Gaussian to exponential crossover in the attenuation of polarization echoes in NMR, Mol. Phys. 95, 1229 (1998).

[22] M. Gärttner, J. G. Bohnet, A. Safavi-Naini, M. L. Wall, J. J. Bollinger, and A. M. Rey, Measuring out-of-timeorder correlations and multiple quantum spectra in a trapped-ion quantum magnet, Nat. Phys. 13, 781 (2017).

[23] K. X. Wei, C. Ramanathan, and P. Cappellaro, Exploring Localization in Nuclear Spin Chains, Phys. Rev. Lett. 120, 070501 (2018).

[24] K. X. Wei, P. Peng, O. Shtanko, I. Marvian, S. Lloyd, C. Ramanathan, and P. Cappellaro, Emergent Prethermalization Signatures in Out-of-Time Ordered Correlations, Phys. Rev. Lett. 123, 090605 (2019).

[25] M. Niknam, L. F. Santos, and D. G. Cory, Sensitivity of quantum information to environment perturbations measured with a nonlocal out-of-time-order correlation function, Phys. Rev. Research 2, 013200 (2020).

[26] A. Widera, S. Trotzky, P. Cheinet, S. Fölling, F. Gerbier, I. Bloch, V. Gritsev, M. D. Lukin, and E. Demler, Quantum Spin Dynamics of Mode-Squeezed Luttinger Liquids in Two-Component Atomic Gases, Phys. Rev. Lett. 100, 140401 (2008).

[27] F. M. Cucchietti, Time reversal in an optical lattice, J. Opt. Soc. Am. B 27, A30 (2010).

[28] C. Weiss, Effective time-reversal via periodic shaking, J. Phys. Conf. Ser. 414, 012032 (2013).

[29] P. Reimann, Foundations of statistical mechanics under experimentally realistic conditions, Phys. Rev. Lett. 101, 190403 (2008).

[30] N. Linden, S. Popescu, A. J. Short, and A. Winter, Quantum mechanical evolution towards thermal equilibrium, Phys. Rev. E 79, 061103 (2009).

[31] A. J. Short, T. C. Farrelly, Quantum equilibration in finite time, New J. Phys. 14, 013063 (2012).

[32] P. Reimann and L. Dabelow, Typicality of prethermalization, Phys. Rev. Lett. 122, 080603 (2019).

[33] P. Reimann, Transportless equilibration in isolated many-body quantum systems, New J. Phys. 21, 053014 (2019).

[34] S. Genway, A. F. Ho, and D. K. K. Lee, Thermalization of local observables in small Hubbard lattices, Phys. Rev. A 86, 023609 (2012).

[35] W. Beugeling, R. Moessner, and M. Haque, Off-diagonal 
matrix elements of local operators in many-body quantum systems, Phys. Rev. E 91, 012144 (2015).

[36] N. P. Konstantinidis, Thermalization away from integrability and the role of operator off-diagonal elements, Phys. Rev. E 91, 052111 (2015).

[37] F. Borgonovi, F. M. Izrailev, L. F. Santos, and V. G. Zelevinsky, Quantum chaos and thermalization in isolated systems of interacting particles, Phys. Rep. 626, 1 (2016).

[38] D. Jansen, J. Stolpp, L. Vidmar, and F. HeidrichMeisner, Eigenstate thermalization and quantum chaos in the Holstein polaron model, Phys. Rev. B 99, 155130 (2019).

[39] L. Dabelow and P. Reimann, Relaxation theory for perturbed many-body quantum systems versus numerics and experiment, Phys. Rev. Lett. 124, 120602 (2020).

[40] E. J. Torres-Herrera and L. F. Santos, Quench dynamics of isolated many-body systems, Phys. Rev. A 89, 043620 (2014).

[41] J. M. Deutsch, Quantum statistical mechanics in a closed system, Phys. Rev. A 43, 2046 (1991).

[42] P. Reimann, Eigenstate thermalization: Deutsch's approach and beyond, New J. Phys. 17, 055025 (2015).

[43] C. Gogolin and J. Eisert, Equilibration, thermalization, and the emergence of statistical mechanics in closed quantum systems, Rep. Prog. Phys. 79, 056001 (2016).

[44] C. Nation and D. Porras, Off-diagonal observable elements from random matrix theory: distributions, fluctuations, and eigenstate thermalization, New. J. Phys. 20, 103003 (2018).

[45] L. D'Alessio, Y. Kafri, A. Polkovnikov, and M. Rigol, From Quantum Chaos and Eigenstate Thermalization to Statistical Mechanics and Thermodynamics, Adv. Phys. 65, 239 (2016).

[46] G. Biroli, C. Kollath, and A. M. Läuchli, Effect of rare fluctuations on the thermalization of isolated quantum systems, Phys. Rev. Lett. 105, 250401 (2010).

[47] T. N. Ikeda, Y. Watanabe, and M. Ueda, Finite-size scaling analysis of the eigenstate thermalization hypothesis in a one-dimensional interacting Bose gas, Phys. Rev. E 87, 012125 (2013).

[48] V. Alba, Eigenstate thermalization hypothesis and integrability in quantum spin chains, Phys. Rev. B 91, 155123 (2015).

[49] Within the experimental error bars, the Bessel-like shape $4 J_{1}(2 x)^{2} /(2 x)^{2}$ is indistinguishable from a Gaussian shape $\exp \left(-x^{2}\right)$. Note that the two functions agree to third order in $x$ and the relative difference of the fourthorder Taylor coefficients is only $1 / 6$. 